\title{
Triplet Leptogenesis in Left-Right Symmetric Seesaw Models
}

\author{
Tomas Hällgren * Thomas Konstandin $\dagger$ and Tommy Ohlsson $\ddagger$ \\ Department of Theoretical Physics, Royal Institute of Technology (KTH), \\ AlbaNova University Center, Roslagstullsbacken 21, 10691 Stockholm, Sweden \\ We discuss scalar triplet leptogenesis in a specific left-right symmetric seesaw \\ model. We show that the Majorana phases that are present in the model can be \\ effectively used to saturate the existing upper limit on the CP-asymmetry of the \\ triplets. We solve the relevant Boltzmann equations and analyze the viability of \\ triplet leptogenesis. It is known for this kind of scenario that the efficiency of lep- \\ togenesis is maximal if there exists a hierarchy between the branching ratios of the \\ triplet decays into leptons and Higgs particles. We show that triplet leptogene- \\ sis typically favors branching ratios with not too strong hierarchies, since maximal \\ efficiency can only be obtained at the expense of suppressed CP-asymmetries.
}

\section{INTRODUCTION}

Today, one of the major challenges of particle physics and cosmology is to find a convincing model, which accounts for the observed baryon asymmetry of the Universe. The baryon-tophoton ratio is observed to be [1]

$$
\eta_{B}=\frac{n_{B}-n_{\bar{B}}}{n_{\gamma}}=(6.1 \pm 0.2) \times 10^{-10}
$$

The introduction of superheavy right-handed neutrinos may account for the baryon asymmetry by the baryogenesis via leptogenesis mechanism [2], whereby the decays of the heavy neutrinos induce a lepton asymmetry, which is then partly converted into a baryon asymmetry by sphaleron processes. This mechanism has the advantage of simultaneously accounting for the smallness of light neutrino masses via the seesaw mechanism [3, 4, 5, 6, 7, 8, 9, 10, 11].

*tomashal@kth.se

tkonstand@kth.se

†tommy@theophys.kth.se 
Recently, it was shown in refs. [12, 13] that in left-right symmetric seesaw models, there exists an eight-fold degeneracy in the right-handed neutrino sector, i.e., for a given lowenergy neutrino phenomenology, the seesaw formula can be inverted to yield eight possible solutions for the triplet Yukawa coupling matrices. It was then demonstrated in refs. [14, 15] that by using fine-tuning and viability of leptogenesis as criteria, it is possible to discriminate among the different solutions. Another nice feature of this model [14] is that the additional Majorana phases can be used to easily saturate the existing upper bounds on the CP-asymmetry of the heavy right-handed neutrinos derived in ref. [16]. Besides, left-right symmetric models do not contain many more parameters than the minimal seesaw type I model, but can be naturally embedded into grand unified theories (GUTs), since a hierarchical Yukawa coupling does not necessarily lead to a fine-tuning problem in the right-handed neutrino sector [14]. In these studies, it was assumed that leptogenesis is solely driven by the decay of the lightest right-handed neutrino.

In the present paper, we study the impact of leptogenesis induced by the decay of the scalar $S U(2)_{L}$ triplet, which appears naturally in left-right symmetric seesaw models. The common lore about triplet leptogenesis is that it is far less efficient than leptogenesis driven by right-handed neutrino decay, since the triplet can annihilate via gauge interactions. This picture was challenged in ref. [17], where the full set of Boltzmann equations for this model were investigated and it was shown that, due to the interplay of the two possible decay channels of the triplet, the efficiency $\eta$ can be quasi-maximal. In addition, an upper bound on the CP-asymmetry as well as a lower limit on the triplet mass were derived in this work in a model-independent seesaw type I+II setting assuming hierarchical light neutrino masses:

$$
M_{T}>2.8 \times 10^{10} \mathrm{GeV}\left(\tilde{m}_{T}=0.001 \mathrm{eV}\right), \quad M_{T}>1.3 \times 10^{11} \mathrm{GeV}\left(\tilde{m}_{T}=0.05 \mathrm{eV}\right) .
$$

In the present work, we will demonstrate that in the left-right symmetric seesaw model the bound on the CP-asymmetry of the triplet decay rate given in ref. [17] can be approximately saturated. This is due to the presence of additional Majorana phases that, compared to the pure type I seesaw model, provides an additional source of $\mathrm{CP}$ violation. Besides, we solve the relevant Boltzmann equations and determine the produced baryon asymmetry. We discuss in detail the occurrence of quasi-maximal efficiency of leptogenesis in the case of a hierarchy in the branching ratios of triplet decays into leptons and Higgs particles, $B_{L} \ll B_{H}$ or $B_{L} \gg B_{H}$. In principle, this effect arises because in the decay channel 
with small branching ratio washout is negligible. This implies that the large efficiency is obtained at the expense of a suppression in the CP-asymmetry that is normalized to the total decay width. These two effects compete with each other and we demonstrate that leptogenesis in fact favors the region in parameter space in which the rate of triplet decays and annihilations are comparable, $\gamma_{A} \approx \gamma_{D}$ at $M_{T} \approx T$, and also the branching ratios are of similar size, $B_{L} \approx B_{H}$.

The paper is organized as follows. In section [I] we present the model and set up the notation. In section [II] we discuss triplet leptogenesis, and in section [V] we draw our conclusions.

\section{LEFT-RIGHT SYMMETRIC MODEL}

In this section, the left-right symmetric model of refs. [14, 17] is presented. The Lagrangian contains the following relevant terms

$$
\begin{aligned}
\mathcal{L} \supset & -\frac{1}{2} M_{T}^{2} \operatorname{tr}\left(T_{L}^{\dagger} T_{L}\right)+\frac{1}{2} \kappa_{i j} \operatorname{tr}\left(T_{R}^{\dagger} \Phi_{i} T_{L} \Phi_{j}^{\dagger}\right) \\
& -\frac{1}{2} f^{\alpha \beta} L_{\alpha}^{T} C \mathrm{i} \tau_{2} T_{L} L_{\beta}-\frac{1}{2} f^{\alpha \beta} R_{\alpha}^{T} C \mathrm{i} \tau_{2} T_{R} R_{\beta} \\
& +y_{i}^{\alpha \beta} \bar{R}_{\alpha} \Phi_{i} L_{\beta}+\text { h.c. }
\end{aligned}
$$

where $L$ and $R$ denote the left- and right-handed leptons, respectively. The Yukawa coupling is assumed to be complex symmetric, i.e., $y_{i}=y_{i}^{T}$, what holds true in certain left-right symmetric GUTs. The Higgs bidoublet fields $\Phi_{i}$ are given by

$$
\Phi_{1}=\left(\begin{array}{cc}
\Phi_{1}^{0} & \Phi_{1}^{+} \\
\Phi_{2}^{-} & \Phi_{2}^{0}
\end{array}\right), \quad \Phi_{2}=\tau_{2} \Phi_{1}^{*} \tau_{2},
$$

and the triplets $T_{L / R}$ can be written as

$$
T_{L / R}=\left(\begin{array}{cc}
T^{+} / \sqrt{2} & T^{++} \\
T^{0} & -T^{+} / \sqrt{2}
\end{array}\right)_{L / R} .
$$

By spontaneous symmetry breaking, the Higgs fields acquire vacuum expectation values (vevs) that are related by

$$
\begin{aligned}
v_{L} & =\frac{v_{R}}{2 M_{T}^{2}} \kappa v^{2}, \\
\kappa v^{2} & =\kappa_{11} v_{2} v_{1}^{*}+\kappa_{12} v_{2}^{2}+\kappa_{21} v_{1}^{* 2}+\kappa_{22} v_{1}^{*} v_{2},
\end{aligned}
$$


where $v_{L / R}$ denote the vevs of the $S U(2)_{L / R}$ scalar triplets $T_{L / R}, v^{2}=\left|v_{1}^{2}\right|+\left|v_{2}^{2}\right| \simeq$ $(174 \mathrm{GeV})^{2}$, and $v_{i}=\left\langle\Phi_{i}^{0}\right\rangle$ are the vevs of the bidoublet.

The resulting neutrino masses lead to the following seesaw formula in the left-right symmetric model

$$
m_{\nu}=m_{T}+m_{H}=f v_{L}-\frac{v^{2}}{v_{R}} y f^{-1} y .
$$

The contribution $m_{H}$ from the heavy right-handed neutrinos is hereby usually called the type I term, while the contribution $m_{T}$ from the triplet is called the type II term. This equation can be simplified by using the fact that the combination $m_{T}=f v_{L}$ depends on the parameter

$$
\mu=\frac{v_{R}}{v_{L} v^{2}}
$$

only and not on $v_{L}$ and $v_{R}$ separately. Given a light neutrino mass matrix $m_{\nu}$ and Yukawa coupling matrix $y$, there exists for $n$ flavors $2^{n}$ solutions for the triplet Yukawa coupling matrix $f$ as shown in refs. [12, 13]. In the case of one flavor, the inversion becomes particularly simple and one finds the following two solutions

$$
f_{ \pm}=\frac{m_{\nu}}{2 v_{L}} \pm \sqrt{\frac{m_{\nu}^{2}}{4 v_{L}^{2}}+\frac{v^{2}}{v_{L} v_{R}} y^{2}} .
$$

An analytical expression for the inversion of the seesaw relation in the three-flavor case is also given in refs. [12, 13]. The properties of these solutions have been studied in refs. [13, 14, 15], and in particular, the eigenvalues and mixing properties were investigated. As in these works, we choose the Yukawa coupling matrix $y$ equal to the up-type quark Yukawa coupling matrix, which can be motivated by GUTs [18, 19, 20]. To be specific, we implement the relation $y=y_{\text {up }}$ at the GUT scale and in the flavor basis. The CKM matrix is completely attributed to the up-quark Yukawa matrix. We utilize the best-fit values for the parameters of the neutrino mass matrix as given in refs. [21, 22, 23]. It is known that depending on the spectrum of the light neutrinos, running effects can also be sizable in the neutrino sector. In the present study we neglect these effects, since the specific values of the mixing angles will not strongly influence our results concerning leptogenesis. This is due to the fact that our main source of CP violation stems from the additional Majorana phases and not from the Dirac phases in the mixing matrices. As additional parameters of the model enter then the five Majorana phases, the mass of the lightest neutrino $m_{0}$, the hierarchy (normal/inverted) of the light neutrinos, and the ratio $v_{R} / v_{L}$. 

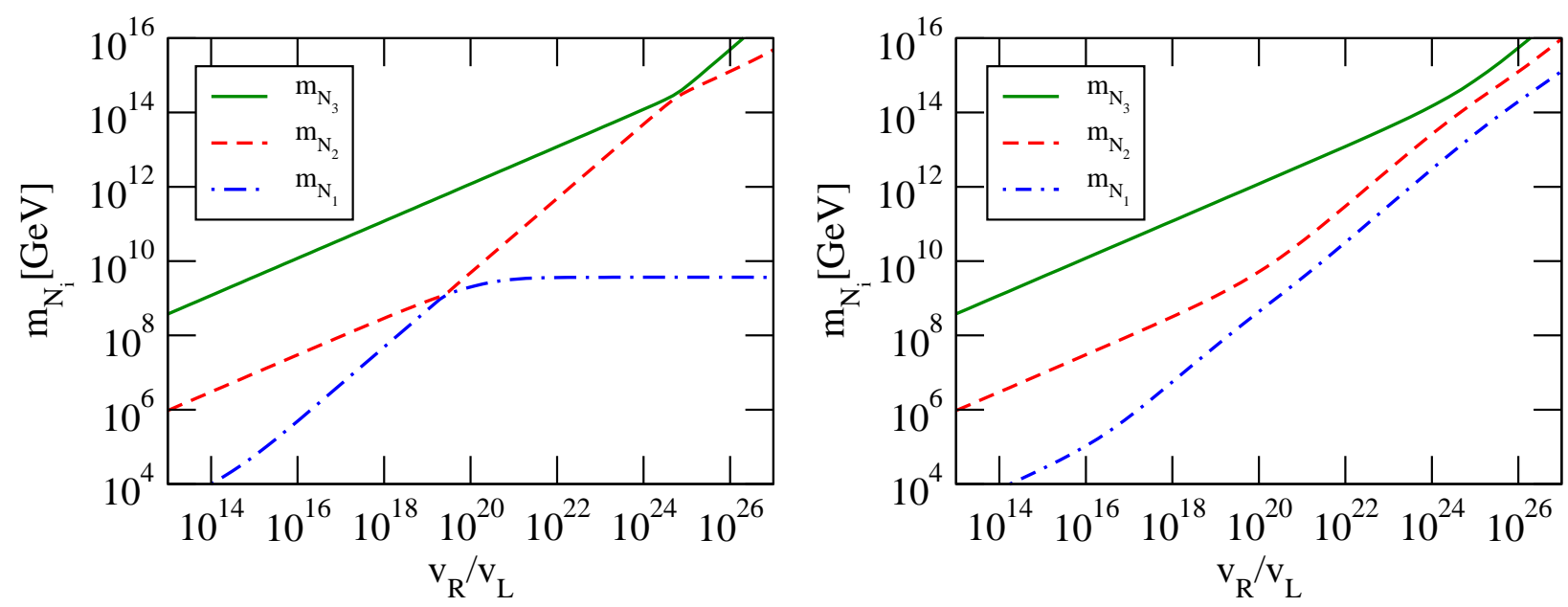

FIG. 1: Right-handed neutrino masses as functions of $v_{R} / v_{L}$ for the two solutions ' +-+ ' (left plot) and ' +++ ' (right plot).

Before we discuss triplet leptogenesis, we remark on some of the general properties of the solutions for the right-handed neutrino masses. In the regime of small $v_{R} / v_{L}$, both contributions in eq. (8) are large and the light neutrino mass matrix results from a precise cancellation of both contributions. This requires a large amount of fine-tuning, and hence constitutes a disfavored region in parameter space. In the regime of large $v_{R} / v_{L}$, the solutions can be labeled according to whether the eigenvalues are type I ('-') or type II ('+') dominated. This corresponds to the choice of the different signs appearing in eq. (10) and results in the eight different solutions in the case of three flavors. If an eigenvalue is type I dominated, it approaches a constant value for large $v_{R} / v_{L}$, which is given by the corresponding eigenvalue of the Yukawa coupling matrix $y$. Thus, the smallness of the up-quark Yukawa coupling leads to the fact that the four solutions of type ' \pm \pm- ' predict a very light right-handed neutrino with a mass below $10^{6} \mathrm{GeV}$. These solutions are disfavored from the point of view of leptogenesis. For the two other solutions of type ' \pm-+ ', the lightest mass instead approaches $10^{9} \mathrm{GeV}$ in the large $v_{R} / v_{L}$ limit, which may induce viable leptogenesis, while being consistent with the bounds on the reheating temperature from gravitino physics as described in detail in ref. [14]. The remaining two solutions predict larger values for the right-handed neutrino masses in the large $v_{R} / v_{L}$ regime. These facts are demonstrated in Fig. 1, where the masses of the right-handed neutrinos, $m_{N} \propto f v_{R}$, for the two solutions ' +-+ ' and ' +++ ' are displayed. 


\section{TRIPLET LEPTOGENESIS}

In the traditional framework of leptogenesis, the CP-asymmetry is induced by the decay of the lightest right-handed neutrino. Here we give a brief review of the case when instead the triplet is the source of leptogenesis. In this case, the CP-asymmetry has been studied in refs. [24, 25, 26]. The full set of Boltzmann equations were derived and solved in ref. [17]. Generally, one would expect that the produced baryon asymmetry is suppressed, since the gauge scattering keeps the triplets close to thermal equilibrium, preventing sufficient out-of-

equilibrium decays. However, it was shown in ref. [17] that, due to an interplay of the two possible decay channels of the triplet, a quasi maximal efficiency can be achieved.

The Lagrangian in ref. [17] contains the following relevant terms

$$
\mathcal{L} \supset-\frac{1}{2} M_{T}^{2} \operatorname{tr}\left(T_{L}^{\dagger} T_{L}\right)-\frac{1}{2} \lambda_{L}^{\alpha \beta} L_{\alpha}^{T} C \mathrm{i} \tau_{2} T_{L} L_{\beta}+\frac{1}{2} \lambda_{H} M_{T} H^{T} \mathrm{i} \tau_{2} T_{L}^{\dagger} H+\text { h.c. }
$$

Comparing this with the Lagrangian in eq. (3), one can make the following two identifications [26]

$$
\lambda_{H} M_{T}=\kappa v_{R}, \quad \lambda_{L}=f .
$$

Notice that perturbative unitarity in the left-right symmetric model implies $\kappa \lesssim 1$ instead of $\lambda_{H} \lesssim 1$ as used in ref. [17]. The former constraint is less severe, since $f \lesssim 1$, and hence, $v_{R} \gtrsim m_{N_{3}} \gtrsim M_{T}$ in the parameter region that is interesting for triplet leptogenesis.

\section{A. CP-asymmetry}

The CP-asymmetry is due to the decay of the triplet via the two possible channels $T_{L} \rightarrow$ $H H$ and $T_{L} \rightarrow \bar{L} \bar{L}$, where $H$ and $L$ denote Higgs and lepton doublets, respectively. We follow the notation of ref. [17] and denote the branching ratio into Higgs bosons by $B_{H}$ and the branching ratio into leptons by $B_{L}$, and assume that these are the only possible decay channels, i.e., $B_{L}+B_{H}=1$. At tree level, the decay rates are given by [17]

$$
\begin{aligned}
\Gamma(T \rightarrow \bar{L} \bar{L}) & =B_{L} \Gamma_{T}=\frac{M_{T}}{16 \pi} \operatorname{tr}\left(\lambda_{L} \lambda_{L}^{\dagger}\right), \\
\Gamma(T \rightarrow H H) & =B_{H} \Gamma_{T}=\frac{M_{T}}{16 \pi} \lambda_{H} \lambda_{H}^{\dagger} .
\end{aligned}
$$


If the triplet is lighter than the right-handed neutrinos, the CP-asymmetry takes the form [17]

$$
\epsilon_{L}=\frac{M_{T}}{4 \pi v^{2}} \sqrt{B_{L} B_{H}} \frac{\operatorname{Im}\left[\operatorname{tr}\left(m_{T}^{\dagger} m_{H}\right)\right]}{\tilde{m}_{T}} .
$$

In this expression, $m_{T}\left(m_{H}\right)$ denotes the triplet (right-handed neutrino) contribution to the light neutrino mass as given in eq. (8). In addition, we have introduced the parameter

$$
\tilde{m}_{T}=\sqrt{\operatorname{tr}\left(m_{T}^{\dagger} m_{T}\right)}
$$

Notice that using eqs. (61) and (12) $m_{T}$ can be recast as

$$
m_{T}=f v_{L}=\lambda_{L} \lambda_{H} \frac{v^{2}}{2 M_{T}}
$$

such that

$$
\Gamma_{T}=\frac{\tilde{m}_{T}}{\sqrt{B_{L} B_{H}}} \frac{M_{T}^{2}}{8 \pi v^{2}} .
$$

An upper bound on the CP-asymmetry was also derived in ref. [17] and found to be

$$
\left|\epsilon_{L}\right| \leq \frac{M_{T}}{4 \pi v^{2}} \sqrt{B_{L} B_{H} \sum_{i} m_{\nu_{i}}^{2}}=: \epsilon_{L, \max }
$$

where $m_{\nu_{i}}$ denote the light neutrino masses.

In our specific model, we use the following input parameters. As mentioned in the last section, a reasonable choice for the Yukawa coupling matrix $y$, motivated by GUTs, is the up-quark Yukawa coupling. In addition, the Majorana phases, the ratio $v_{R} / v_{L}$, the lightest neutrino mass $m_{0}$, and the hierarchy of the light neutrinos have to be specified. Using these parameters, the seesaw formula in eq. (8) can be used to determine $f v_{L}$, and thus, the masses of the right-handed neutrinos and the parameter $\tilde{m}_{T}$. The CP-asymmetry $\epsilon_{L}$ depends in addition on $B_{L}$ and $B_{H}$, but only through the explicit factor in eq. (15).

The values of $B_{L}$ and $B_{H}$ depend in the case of fixed $v_{R} / v_{L}$ on the choice of $v_{R}$ or $v_{L}$ according to

$$
\frac{B_{L}}{B_{H}}=\frac{\operatorname{tr}\left(\lambda_{L} \lambda_{L}^{\dagger}\right)}{\lambda_{H} \lambda_{H}^{\dagger}}=\frac{\sum_{i} m_{N_{i}}^{2}}{4 v_{R}^{2} v_{L}^{2} M_{T}^{2}} v^{4} .
$$

In the following, we plot the quantities $\tilde{m}_{T}$ and $\epsilon_{L} / \epsilon_{L, \max }$ that are both independent from the choice of $B_{H}, B_{L}$, and $M_{T}$. Figure 2 shows that it is approximately possible to saturate the bound on the CP-asymmetry stated in eq. (19) for a certain choice of the Majorana 

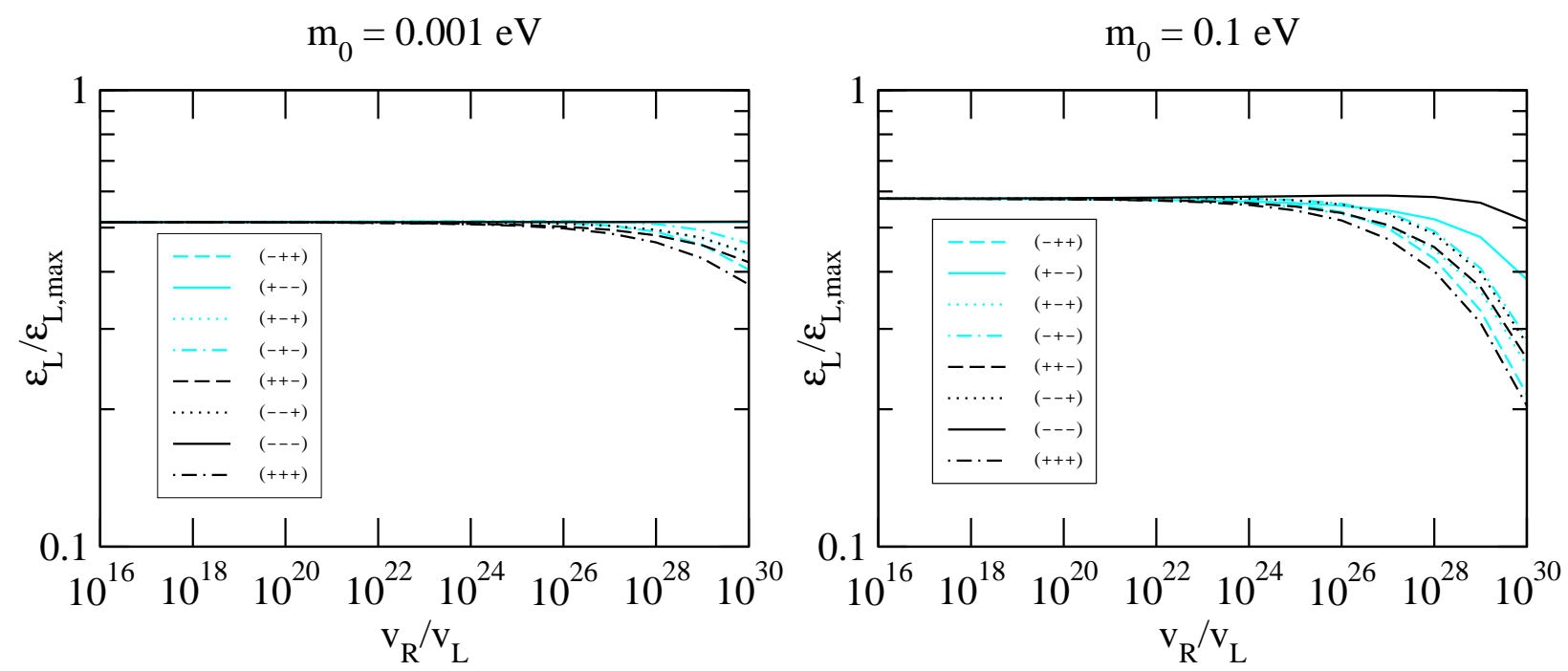

FIG. 2: The parameter $\epsilon_{L} / \epsilon_{L, \max }$ for all eight solutions as a function of $v_{R} / v_{L}$. Inverted hierarchy and $m_{0}=0.001 \mathrm{eV}\left(m_{0}=0.1 \mathrm{eV}\right)$ in the left (right) plot.
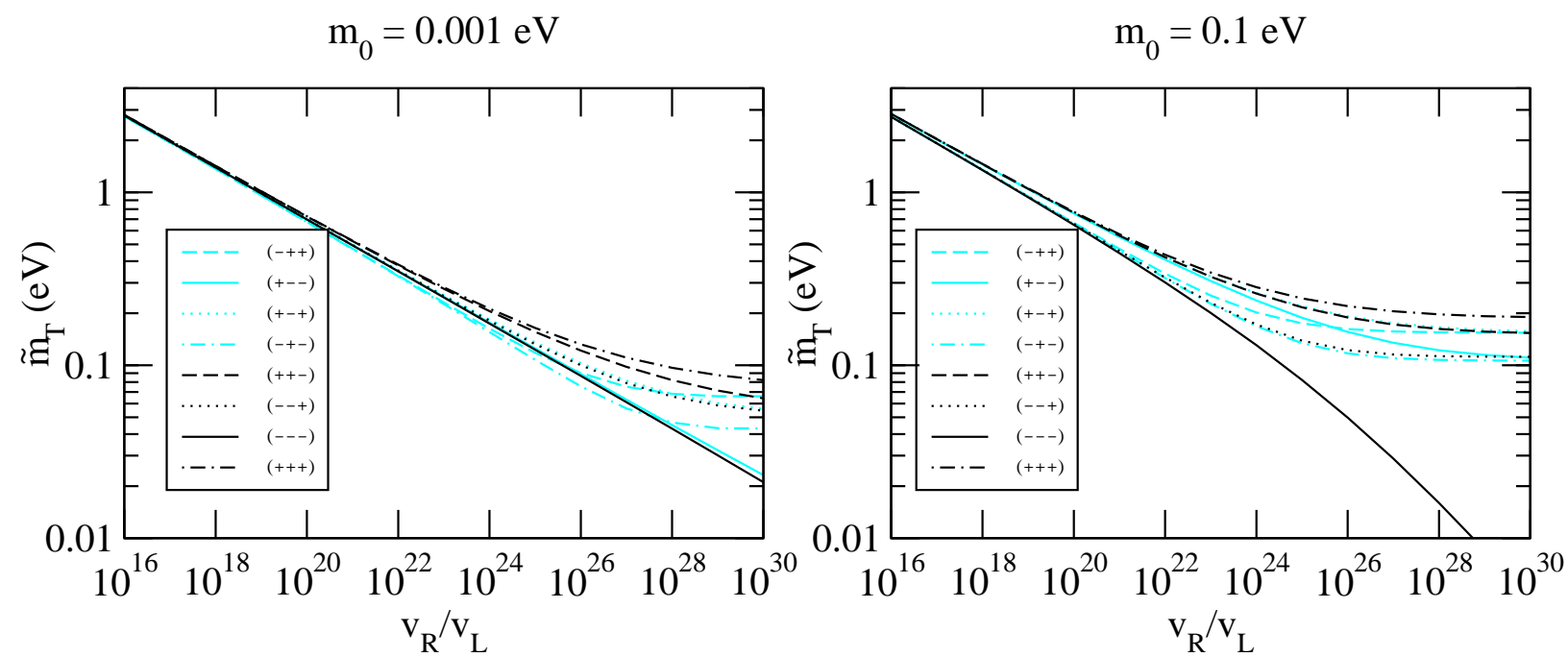

FIG. 3: The parameter $\tilde{m}_{T}$ in units of eV for all eight solutions as a function of $v_{R} / v_{L}$. Inverted hierarchy and $m_{0}=0.001 \mathrm{eV}\left(m_{0}=0.1 \mathrm{eV}\right)$ in the left (right) plot.

phases. Additionally, $\epsilon_{L} / \epsilon_{L \text {, max }}$ does not depend on $v_{R} / v_{L}$ up to $v_{R} / v_{L} \approx 10^{28}$. On the other hand, $\tilde{m}_{T}$ generally decreases for increasing $v_{R} / v_{L}$, as shown in Fig. 3 ,

Let us summarize our findings in the left-right symmetric model concerning triplet leptogenesis: One main parameter in the model is the ratio $v_{R} / v_{L}$ that also enters into the masses of the right-handed neutrinos. Efficient triplet leptogenesis requires the lightest right-handed neutrino to be heavier than the triplet, otherwise inverse decays into the neutrino tend to erase every lepton asymmetry produced by triplet decays. In the light of the constraint in 
eq. (2) and Fig. 11, this is only the case for the two solution of type ' \pm++ ' and $v_{R} / v_{L} \gtrsim 10^{22}$. The CP-asymmetry of the triplet does not vary strongly as long as $v_{R} / v_{L} \lesssim 10^{28}$ and for an appropriate choice for the Majorana phases, the upper bound given in eq. (19) can be approximately saturated. This upper bound is proportional to $\sqrt{\sum_{i} m_{\nu_{i}}^{2}}$ such that large values of $m_{0}$ seem to be opportune for triplet leptogenesis. On the other hand, the effective mass parameter $\tilde{m}_{T}$ that will enter in the Boltzmann equations seems to be constrained by $\tilde{m}_{T} \gtrsim m_{0}$ such that larger values of $m_{0}$ might lead to larger washout. This question will be discussed in the next section. The value of $\tilde{m}_{T}$ can in a certain range be chosen by specifying the ratio $v_{R} / v_{L}$. On the other hand, the parameters $B_{L}$ and $B_{H}$ can be adjusted by changing $v_{R}$ or $v_{L}$ for fixed $v_{R} / v_{L}$ according to eq. (20). Hence, we will treat $B_{L}\left(B_{H}\right)$, $M_{T}$, and $\tilde{m}_{T}$ as free parameters in the following discussion of the transport equations.

\section{B. Boltzmann equations}

The complete set of Boltzmann equations has been derived in ref. [17] and we will discuss some qualitative results in the following. First, since triplets, different from Majorana neutrinos, are not self-conjugated fields, the evolution of the number asymmetry $n_{T}-n_{\bar{T}}$ is governed by an additional equation. Besides, the system of equations describes the evolution of the number density of the Higgs particles $n_{H}$. The rates that are most important for determining the efficiency $\eta$ are the gauge scatterings $\gamma_{A}$ and the triplet decays into Higgs bosons $\left(\gamma_{H}=B_{H} \gamma_{D}\right)$ and leptons $\left(\gamma_{L}=B_{L} \gamma_{D}\right)$. If one of the decay rates of the triplet $\left(\gamma_{H}\right.$ or $\left.\gamma_{L}\right)$ is larger than the gauge scatterings, the triplets decay before annihilating, thus making the gauge scatterings inefficient. This would also imply that the triplet decay rate is larger than the expansion rate, which would therefore seem to make leptogenesis impossible. However, if the second decay rate is smaller than the expansion rate, a lepton asymmetry can be induced. It is this interplay of $\gamma_{L}, \gamma_{H}$, and $\gamma_{A}$, which can result in a quasi-maximal efficiency in triplet leptogenesis, as demonstrated in ref. [17]. On the other hand, a hierarchy between $B_{L}$ and $B_{H}$ leads to a suppression in the CP-asymmetry as can be seen in eq. (19) such that it is not guaranteed that the produced baryon asymmetry is maximal in this region of the parameter space.

Our results are obtained by solving a simplified set of transport equations, where we include the $\Delta T=2$ scatterings as given in ref. [17], but neglect the $\Delta L=2$ scatterings that 
are minute in most cases. The corresponding set of Boltzmann equations are $\left(z=M_{T} / T\right)$

$$
\begin{aligned}
& s H z \frac{d \Sigma_{T}}{d z}=-\gamma_{D}\left(\frac{\Sigma_{T}}{\Sigma_{T}^{e q}}-1\right)-2 \gamma_{A}\left(\frac{\Sigma_{T}^{2}}{\Sigma_{T}^{2 e q}}-1\right) \\
& s H z \frac{d \Delta_{L}}{d z}=\epsilon_{L} \gamma_{D}\left(\frac{\Sigma_{T}}{\Sigma_{T}^{e q}}-1\right)-2 \gamma_{D} B_{L}\left(\frac{\Delta_{L}}{Y_{L}^{e q}}+\frac{\Delta_{T}}{\Sigma_{T}^{e q}}\right), \\
& s H z \frac{d \Delta_{H}}{d z}=\epsilon_{L} \gamma_{D}\left(\frac{\Sigma_{T}}{\Sigma_{T}^{e q}}-1\right)-2 \gamma_{D} B_{H}\left(\frac{\Delta_{H}}{Y_{H}^{e q}}-\frac{\Delta_{T}}{\Sigma_{T}^{e q}}\right), \\
& s H z \frac{d \Delta_{T}}{d z}=-\gamma_{D}\left(\frac{\Delta_{T}}{\Sigma_{T}^{e q}}+B_{L} \frac{\Delta_{L}}{Y_{L}^{e q}}-B_{H} \frac{\Delta_{H}}{Y_{H}^{e q}}\right)
\end{aligned}
$$

where $\Sigma_{T}, \Delta_{X}$, and $Y_{X}$ denote particle numbers normalized to the entropy

$$
\Sigma_{T}=\left(n_{T}+n_{\bar{T}}\right) / s, \quad \Delta_{X}=\left(n_{X}-n_{\bar{X}}\right) / s, \quad Y_{X}=n_{X} / s
$$

that are in equilibrium given by

$$
s=g_{*} \frac{2 \pi^{2}}{45} T^{3}, \quad n_{\gamma}=2 \frac{\zeta(3)}{\pi^{2}} T^{3}, \quad n_{L}^{e q}=\frac{27}{4} n_{\gamma}, \quad n_{H}^{e q}=2 n_{\gamma}, \quad n_{T}^{e q}=\frac{3}{4} n_{\gamma} z^{2} K_{2}(z) .
$$

The Hubble parameter is $H \simeq 1.66 \sqrt{g_{*}} T^{2} / M_{P l}$ and the rate of triplet decays and inverse decays is given by

$$
\gamma_{D}=s \Gamma_{T} \Sigma_{T}^{e q} K_{1}(z) / K_{2}(z)
$$

where $\Gamma_{T}$ is the decay width as given in eq. (13). The annihilation rate $\gamma_{A}$ turns out to be

$$
\begin{aligned}
\gamma_{A}(z)= & \frac{T M_{T}^{3}}{64 \pi^{4}} \int_{4}^{\infty} d x \sqrt{x} K_{1}(z \sqrt{x}) \hat{\sigma}(x), \\
\hat{\sigma}(x)= & \frac{50 g_{2}^{4}+41 g_{Y}^{2}}{16 \pi} r^{3}+\frac{r}{2 \pi}\left[g_{2}^{4}(10+68 / x)+g_{Y}^{4}(1+4 / x)\right] \\
& +\frac{1}{2 \pi x^{2}} \log \frac{1+r}{1-r}\left[g_{2}^{4}(48 x-48)+g_{Y}^{4}(12 x-24)\right]
\end{aligned}
$$

with $r=\sqrt{1-4 / x}$ and $g_{2}\left(g_{Y}\right)$ denotes the $S U(2)_{L}\left(U(1)_{Y}\right)$ gauge coupling of the Standard Model. The efficiency $\eta$ is defined by

$$
\Delta_{L}=\left.\epsilon_{L} \eta \Sigma_{T}\right|_{T \gg M_{T}}
$$

such that the baryon-to-photon ratio is at late times given by

$$
\eta_{B}=\frac{n_{B}}{n_{\gamma}}=-\frac{28}{79} \frac{s}{n_{\gamma}} \Delta_{L} \simeq-0.039 \epsilon_{L} \eta .
$$

Before we present numerical results, we analyze the qualitative behavior of the solutions to the transport equations in certain limiting cases. First of all, notice that eq. (21) that described the dynamics of the total triplet number density decouples from the other equations. 
An explicit solution is given by

$$
\begin{aligned}
\mathcal{S}(z) & =\frac{\gamma_{D}}{s H}\left(\frac{\Sigma_{T}}{\Sigma_{T}^{e q}}-1\right) \\
& \approx \frac{\gamma_{D}}{s H \Sigma_{T}^{e q}} \int^{z} d z^{\prime} \frac{d \Sigma_{T}^{e q}\left(z^{\prime}\right)}{d z^{\prime}} \exp \left(-\int_{z^{\prime}}^{z} \frac{d \tilde{z}}{\tilde{z}} \frac{\gamma_{D}+4 \gamma_{A}}{s H \Sigma_{T}^{e q}}(\tilde{z})\right)
\end{aligned}
$$

Here, the function $\mathcal{S}$ was defined that will act as a source for the lepton and Higgs asymmetries. The magnitude of the source depends on the two decay rates that are to high precision given by the approximations

$$
\begin{aligned}
& \frac{\gamma_{D}}{s H} \simeq \frac{1}{\sqrt{B_{L} B_{H}}} \frac{\tilde{m}_{T}}{10^{-3} \mathrm{eV}} z^{4} K_{1}(z) \\
& \frac{\gamma_{A}}{s H} \simeq \frac{10^{14} \mathrm{GeV}}{M_{T}} z^{4} K_{2}(2 z)\left(\frac{1}{1+25 z^{2}}\right)^{1 / 4} .
\end{aligned}
$$

For small values of the triplet mass, $M_{T} \lesssim 10^{11} \mathrm{GeV}$, the annihilation rate is strong enough to keep the triplets close to equilibrium when they become non-relativistic. Hence, the source increases linearly with the decay rate as long as $\gamma_{D} \ll \gamma_{A}$, but becomes exponentially suppressed for $\gamma_{D} \gg \gamma_{A}$. The branching ratio that maximizes the produced baryon asymmetry depends crucially on the question if triplet decays are faster than annihilation processes or vice versa, as will be discussed in the following.

From the three equations that determine the dynamics of the lepton number and Higgs asymmetries, eq. (24) is redundant, since $U(1)_{Y}$ conservation implies that

$$
2 \Delta_{T}=\Delta_{L}-\Delta_{H}
$$

and the remaining two equations are then of the form

$$
\begin{aligned}
z \frac{d \Delta_{L}}{d z} & =\epsilon_{L} \mathcal{S}-2 B_{L} \frac{\gamma_{D}}{H s}\left(\frac{\Delta_{L}}{Y_{L}^{e q}}+\frac{\Delta_{L}-\Delta_{H}}{2 \Sigma_{T}^{e q}}\right) \\
z \frac{d \Delta_{H}}{d z} & =\epsilon_{L} \mathcal{S}-2 B_{H} \frac{\gamma_{D}}{H s}\left(\frac{\Delta_{H}}{Y_{H}^{e q}}-\frac{\Delta_{L}-\Delta_{H}}{2 \Sigma_{T}^{e q}}\right) .
\end{aligned}
$$

Since $\Delta_{T}$ is proportional to $\Delta_{L}-\Delta_{H}$, the effect of eq. (24) is to distribute a generated asymmetry between the two channels. This process is dominant at late times, when all triplets decay and $\Sigma_{T}^{e q} \ll Y_{L / H}$, such that $\Delta_{L}=\Delta_{H}$ for $z \rightarrow \infty$. Thus, it is not important in which channel the asymmetry was generated initially.

First, we discuss the limit $\gamma_{D} \gg \gamma_{A}$ when annihilation processes are negligible. If one of the branching ratios, e.g. $B_{H}$, is much smaller than the other, washout is only operative 

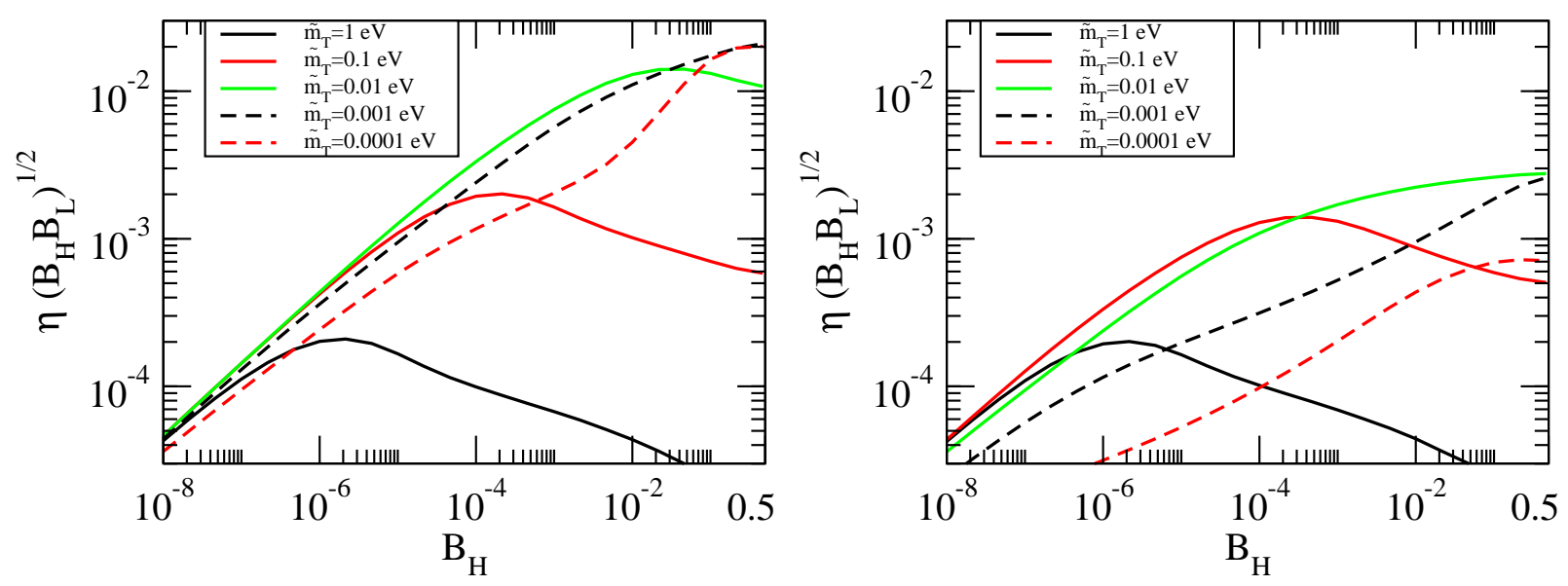

FIG. 4: The quantity $\eta \sqrt{B_{L} B_{H}}$ as a function of $B_{H}$ for $M_{T}=2 \times 10^{12} \mathrm{GeV}$ (left plot) and $M_{T}=2 \times 10^{10} \mathrm{GeV}$ (right plot) and five different values of the parameter $\tilde{m}_{T}$.

in one channel, while the other channel accumulates a significant asymmetry. Nevertheless, this asymmetry is partially reduced by the mixing with the triplet asymmetry $\Delta_{T}$ and finally equally distributed between the two channels. In this regime, the efficiency $\eta$ does not depend strongly on the branching ratios and, comparing with eq. (15), one expects that the produced baryon asymmetry is proportional to $\sqrt{B_{L} B_{H}} \approx \sqrt{B_{H}}$. On the other hand, for larger values of $B_{H}$ washout is significant and the baryon asymmetry produced by the Higgs decay channel starts to be exponentially suppressed for

$$
B_{H} \frac{\gamma_{D}}{H n_{\gamma}} \gtrsim 1
$$

such that according to eq. (34) the optimal choice for $B_{H}$ (and likewise for $B_{L}$ ) scales as

$$
B_{\max } \propto \tilde{m}_{T}^{-2}
$$

This is demonstrated in Fig. 4 that shows $\eta \sqrt{B_{L} B_{H}}$ as a function of $B_{H}$ for $M_{T}=2 \times$ $10^{12} \mathrm{GeV}$ and $M_{T}=2 \times 10^{10} \mathrm{GeV}$ and five different values of $\tilde{m}_{T}$. Notice also that in this limit the annihilation rate and consequently the triplet mass is not relevant for the efficiency $\eta$. Since the branching ratios $B_{L}$ and $B_{H}$ enter in the CP-asymmetry only by the explicit factor in eq. (15), we show in Fig. 4 the combination $\eta \sqrt{B_{L} B_{H}}$ that is proportional to the baryon asymmetry $\eta_{B}$. A specific example for a solution of the transport equations is given in Fig. 5 .

In the opposite limit, $\gamma_{D} \ll \gamma_{A}$, the source $\mathcal{S}$ is proportional to $\gamma_{D}$, according to eq. (32). Washout is not relevant and the efficiency $\eta$ is independent of $B_{L}$ and $B_{H}$ even for large 

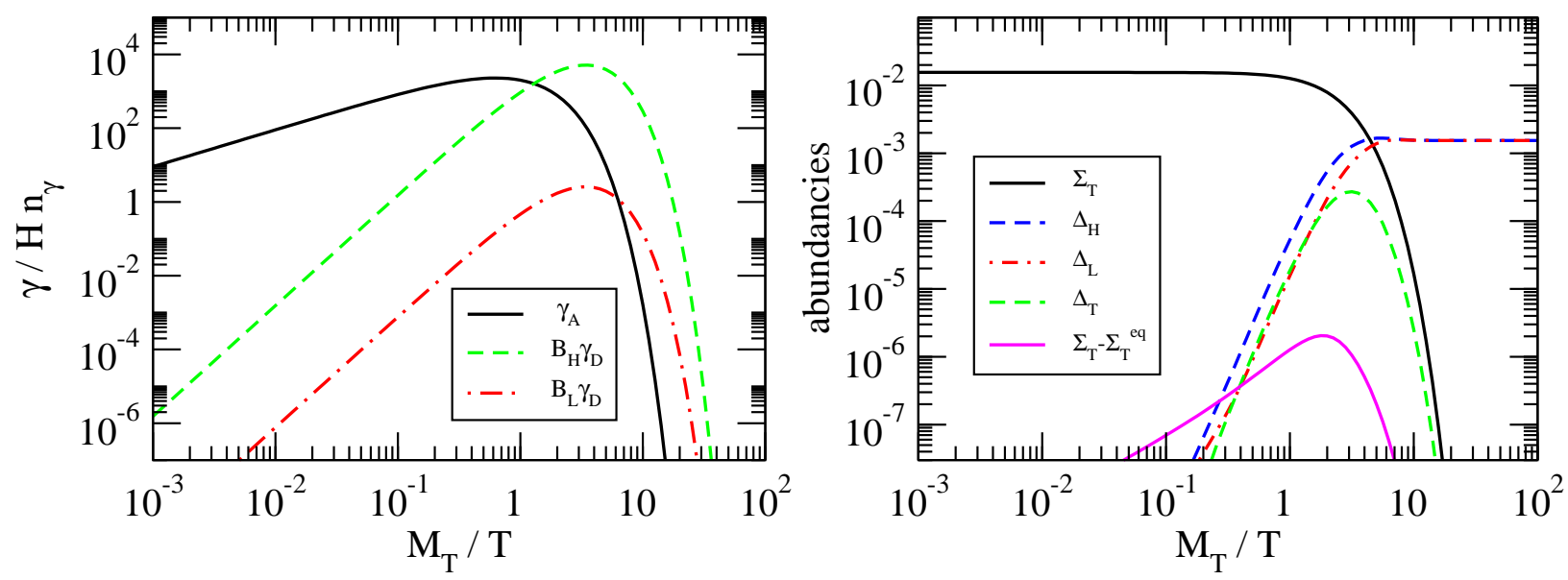

FIG. 5: The left plot shows the decay rates $\gamma_{A}, B_{H} \gamma_{D}$, and $B_{L} \gamma_{D}$ in units of $H n_{\gamma}$. The right plot shows the abundances $\Sigma_{T}, \Delta_{L}, \Delta_{H}, \Delta_{T}$, and $\Sigma_{T}-\Sigma_{T}^{e q}$ for $\epsilon_{L}=1$. The used parameters are $M_{T}=10^{11} \mathrm{GeV}, B_{H}=5 \times 10^{-4}$, and $\tilde{m}_{T}=0.05 \mathrm{eV}$ resulting in $\eta=0.1$.

branching ratios. Hence, the predicted baryon asymmetry is maximal for the choice $B_{L} \approx$ $B_{H} \approx 1 / 2$, since in this case the $\mathrm{CP}$-asymmetry is maximal. This behavior can be seen in Fig. 4. Notice that in the left plot the limit $\gamma_{D} \approx \gamma_{A}$ is barely reached.

With respect to the effective mass $\tilde{m}_{T}$, leptogenesis favors the region in parameter space, where the decay rate and the annihilation rate are comparable, $\gamma_{D} \approx \gamma_{A}$ at $z \approx 1$. In the case of a rather heavy triplet (left plot of Fig. 4), annihilations are suppressed such that the situation is similar to leptogenesis driven by right-handed neutrino decays. Leptogenesis is maximal when the decay rate is small, $\tilde{m}_{T} \lesssim 0.01 \mathrm{eV}$, and also the branching ratios are of similar size, $B_{L} \approx B_{H}$. In the case of a smaller triplet mass (right plot of Fig. 4), annihilations become important and compete with the two decay processes. Still, a large hierarchy between the branching ratios is not required for leptogenesis, even though the optimal value of the branching ratio depends in this region on the specific values of the decay and annihilation rates.

\section{DISCUSSION}

Before we discuss our results, we remind the reader that most of the conclusions drawn in the left-right symmetric seesaw model depend on the fact that the eigenvalues of the Yukawa coupling matrix $y$ contain a large hierarchy. Some statements depend even on the fact that we assume $y$ to be similar to the up-quark mass matrix. On the other hand, we believe this 
to be the natural choice in left-right symmetric models. In seesaw models of pure type I, a large hierarchy in the Yukawa couplings requires a certain amount of fine-tuning, since the Majorana coupling matrix $f$ inherits the doubled hierarchy of $y$. In type I+II models, and particularly in the left-right symmetric model under consideration, this does not hold true so that these models can be more easily embedded into a GUT, what motivates our choice for the Yukawa coupling matrix.

First of all, viable triplet leptogenesis requires that the triplet is lighter than the lightest right-handed neutrino, otherwise inverse decays erase every produced lepton asymmetry. In the present left-right symmetric model, this implies that only the two solutions for the Majorana masses of type ' \pm++ ' can account for the observed baryon asymmetry, and additionally $v_{R} / v_{L} \gtrsim 10^{22}$ is required. Concerning the CP-asymmetry $\epsilon_{L}$, we found that the upper bound presented in ref. [17] can be approximately (up to a factor 2) saturated by utilizing the Majorana phases in the Yukawa coupling matrix $y$. In addition, $\epsilon_{L}$ is nearly constant for values $v_{R} / v_{L}<10^{28}$. Hence, the prospects of triplet leptogenesis are promising in the region

$$
10^{22} \lesssim v_{R} / v_{L} \lesssim 10^{28}
$$

Notice from Fig. 3 that in this regime the parameter $\tilde{m}_{T}$ varies between $m_{0}$ (the lightest left-handed neutrino mass) and a few tenths of $\mathrm{eV}$ such that a judicious choice of $v_{R} / v_{L}$ can be used to specify $\tilde{m}_{T}$ in this region, while the parameter $\epsilon_{L}$ is unaffected. The remaining free parameters in the left-right symmetric model can be used to adjust the branching ratios $B_{L}$ and $B_{H}$. This implies that in the present model the parameters $B_{H}\left(B_{L}\right), \tilde{m}_{T}$, and $M_{T}$ can in certain ranges be considered as independent parameters.

The results for the baryon asymmetry can be qualitatively understood in different limits as follows. For large triplet decay rates, the gauge interactions are irrelevant. In this case, washout is very strong and leads to exponential suppression unless one of the branching ratios is tiny. On the other hand, for small triplet decay rates, inverse decays are not relevant such that the efficiency $\eta$ is almost independent of $B_{L}$ and $B_{H}$. In this regime, the produced baryon asymmetry is, as the CP-asymmetry, proportional to $\sqrt{B_{L} B_{H}}$, and hence, maximized by the choice $B_{L}=B_{H}=1 / 2$. The most promising region for leptogenesis is given be the intermediate regime, $\gamma_{D} \approx \gamma_{A}$ at $z \approx 1$, and $B_{L} \approx B_{H}$, which is shown in Fig. 4. However, this region is not accessible by the left-right symmetric model, since generically $\tilde{m}_{T} \gtrsim 0.05 \mathrm{eV}$, and the triplet decay rate exceeds the annihilation rate for light triplets, 
$M_{T} \lesssim 10^{12} \mathrm{GeV}$. This makes a hierarchy in the branching ratios necessary and we find the lower bound on the triplet mass $M_{T}$ to be given by

$$
M_{T} \gtrsim 1.0 \times 10^{11} \mathrm{GeV}\left(\tilde{m}_{T}=0.05 \mathrm{eV}\right)
$$

With the optimal choice $B_{H}=10^{-3}$, the produced baryon asymmetry is then given by

$$
\begin{aligned}
\eta & =9.1 \times 10^{-2} \\
\epsilon_{L, \max } & =7.2 \times 10^{-7} \frac{m_{0}}{0.05 \mathrm{eV}}, \\
\eta_{B} & =25.7 \times 10^{-10} \frac{m_{0}}{0.05 \mathrm{eV}} \frac{\epsilon_{L}}{\epsilon_{L, \max }},
\end{aligned}
$$

which is in accordance with observation for $m_{0} \approx 0.05 \mathrm{eV}$ and for an almost maximal CPasymmetry. This is slightly better than the result obtained in ref. [17], since we maximized the baryon asymmetry with respect to $B_{H}$. Nevertheless, the prospects of triplet leptogenesis are generally worse than the ones of leptogenesis driven by right-handed neutrino decays in the left-right symmetric model [14] and are not compatible with the bounds on the reheating temperature coming from gravitino physics in supersymmetric models.

\section{Acknowledgments}

This work was supported by the Göran Gustafsson Foundation [T.H. and T.O.], the Swedish Research Council (Vetenskapsrådet), contract nos. 621-2001-1611 [T.K. and T.O.] and 621-2005-3588 [T.O.], and the Royal Swedish Academy of Sciences (KVA) [T.O.].

[1] D. N. Spergel et al. (WMAP Collaboration), Astrophys. J. Suppl. 148, 175 (2003), astroph/0302209.

[2] M. Fukugita and T. Yanagida, Phys. Lett. B174, 45 (1986).

[3] P. Minkowski, Phys. Lett. B67, 421 (1977).

[4] M. Gell-Mann, P. Ramond, and R. Slansky (1980), print-80-0576 (CERN).

[5] T. Yanagida (1979), in Proceedings of the Workshop on the Baryon Number of the Universe and Unified Theories, Tsukuba, Japan, 13-14 Feb 1979.

[6] S. L. Glashow, NATO Adv. Study Inst. Ser. B Phys. 59, 687 (1979). 
[7] R. N. Mohapatra and G. Senjanović, Phys. Rev. Lett. 44, 912 (1980).

[8] M. Magg and C. Wetterich, Phys. Lett. B94, 61 (1980).

[9] G. Lazarides, Q. Shafi, and C. Wetterich, Nucl. Phys. B181, 287 (1981).

[10] J. Schechter and J. W. F. Valle, Phys. Rev. D22, 2227 (1980).

[11] R. N. Mohapatra and G. Senjanović, Phys. Rev. D23, 165 (1981).

[12] E. K. Akhmedov and M. Frigerio, Phys. Rev. Lett. 96, 061802 (2006), hep-ph/0509299.

[13] E. K. Akhmedov and M. Frigerio, JHEP 01, 043 (2007), hep-ph/0609046.

[14] E. K. Akhmedov, M. Blennow, T. Hällgren, T. Konstandin, and T. Ohlsson, JHEP 04, 022 (2007), hep-ph/0612194.

[15] P. Hosteins, S. Lavignac, and C. A. Savoy, Nucl. Phys. B755, 137 (2006), hep-ph/0606078.

[16] S. Antusch and S. F. King, Phys. Lett. B597, 199 (2004), hep-ph/0405093.

[17] T. Hambye, M. Raidal, and A. Strumia, Phys. Lett. B632, 667 (2006), hep-ph/0510008.

[18] J. C. Pati and A. Salam, Phys. Rev. D8, 1240 (1973).

[19] H. Georgi, AIP Conf. Proc. 23, 575 (1975).

[20] H. Fritzsch and P. Minkowski, Ann. Phys. 93, 193 (1975).

[21] M. Maltoni, T. Schwetz, M. A. Tórtola, and J. W. F. Valle, New J. Phys. 6, 122 (2004), hep-ph/0405172.

[22] A. Strumia and F. Vissani, Nucl. Phys. B 726, 294 (2005), hep-ph/0503246.

[23] G. L. Fogli, E. Lisi, A. Marrone, A. Palazzo, and A. M. Rotunno, Prog. Part. Nucl. Phys. 57, $71(2006)$.

[24] U. Sarkar, Phys. Rev. D59, 031301 (1999), hep-ph/9807466.

[25] G. Lazarides, Phys. Lett. B452, 227 (1999), hep-ph/9812454.

[26] T. Hambye and G. Senjanović, Phys. Lett. B582, 73 (2004), hep-ph/0307237. 